\title{
Präkanzerosen und Frühkarzinome des Gastrointestinaltrakts
}

Präkanzerosen und Frühkarzinome des Gastrointestinaltrakts rücken zunehmend in den Mittelpunkt des Interesses von Gastroenterologen und Viszeralchirurgen. Dabei lösen wir uns von der alleinigen Diagnostik mit dem Ziel der Früherkennung von malignen Tumoren und wenden uns mehr und mehr den Problemen und Möglichkeiten minimal invasiver Behandlungstechniken und selbst dem Einsatz präventiver Therapieformen zu.

Spätestens nach der Entdeckung der Adenom-Karzinom-Sequenz ist die endoskopische Entfernung adenomatöser Polypen im Kolon und Rektum ein etabliertes Verfahren. Die medikamentöse Therapie von Adenomen ist derzeit Focus intensiver Forschungsarbeiten. Wird die Entstehung von Karzinomen so zu verhindern sein?

Unsicherheit besteht in der Problematik des gastroösophagealen Refluxes und des Barrett-Ösophagus. Mit diesen Krankheitsbildern sehen wir uns immer häufiger konfrontiert. Wie sind die derzeit eingesetzten medikamentösen Verfahren, wie die verschiedenen Methoden von Antirefluxoperationen und wie die lokal abladierenden Therapiekonzepte langfristig zu bewerten?

Wie radikal muss die Operation bei frühen Formen des Magenkarzinoms sein? Zur Feststellung der intra- und submukösen Tumorausdehnung sowie des Lymphknotenstatus von Magenfrühkarzinomen wurden diagnostische Verfahren entwickelt, die möglicherweise lokale endoskopische und kombinierte endoskopisch-laparoskopische Operationsmethoden erlauben, ohne die definitiven Heilungsraten zu beeinträchtigen. Die Techniken werden zur Zeit evaluiert. Zweifellos würde die Reduzierung der Radikalität unter dieser Zielsetzung zu einer postoperativen Verbesserung der Lebensqualität führen.

Die hepatitisinduzierte Leberzhirrose bedeutet ein hohes Risiko für die Entwicklung hepatozellulärer Karzinome. Ähnliches gilt für die primär biliäre Zirrhose und die sklerosierende
Cholangitis. Wie lässt sich die Tumorentstehung konservativ beherrschen? Welche Patienten profitieren von der chirurgischen Therapie und zu welchem Zeitpunkt bzw. bei welcher Konstellation der Erkrankung ist die operative Resektion oder die Transplantation optimal?

Schließlich sind die Erkenntnisse zu hereditären malignen Tumoren noch sehr im Fluss. Wenn auch die sporadischen Neoplasien im Gastrointestinaltrakt mehr als $90 \%$ aller Malignome betreffen, ist die Gruppe der familiären Formen von großem Interesse. Hier sind inzwischen Mutationen bekannt, die Fragen nach der Vertretbarkeit präventiver Behandlungen aufwerfen. Medikamentöse Ansätze sind Gegenstand intensiver wissenschaftlicher Bearbeitung. Die Proktokolektomie ist bei der familiären Adenomatosis coli anerkannt - am besten noch vor der Entstehung eines Karzinoms und kontinenzerhaltend mit Ileumpouch-analer Anastomose.

Wie ist das hereditäre Nicht-Polyposis-bedingte kolorektale Karzinom (HNPCC) einzustufen? Ist die wiederholte Resektion von im Laufe des Lebens auftretenden metachronen Karzinomen günstiger als eine weitergehende radikale und gleichzeitig präventive Kolektomie? Reicht beim bekannten Mutationsträger eine regelmäßige jährliche Vorsorge zur Verbesserung der Langzeitprognose aus oder sind bereits im genotypischen Stadium allein präventive Maßnahmen möglich oder angezeigt?

Eine Vielzahl von offenen Fragen erfordert die ständige Auseinandersetzung mit diesen Themen, die derzeit nur teilweise zu beantworten sind. Die Entwicklung zu ständig neuen Erkenntnissen ist äußerst dynamisch. In diesem Themenheft stellen ausgewiesene Kenner der verschiedenen pathologischen Entitäten den aktuellen Wissensstand zu diesen Erkrankungen vor. Wir danken ihnen für ihre kompetente Bearbeitung und hoffen auf das Interesse der Leser.

C. Ell, Wiesbaden H.-D. Saeger, Dresden 